\title{
Fate of stem cells grown on the extracellular matrix isolated from cancer cells and their possible applications in tissue engineering
}

\author{
Koyeli Girigoswami*, Devender, N. Srinivasan and Agnishwar Girigoswami \\ Tissue Engineering and Regenerative Medicine Laboratory, Faculty of Allied Health Sciences, Chettinad Hospital and Research Institute, \\ Chettinad Academy of Research and Education, Kelambakkam 603 103, India
}

Propagation of stem cells in abundance is essential for use as cell therapy in regenerative medicine. Proliferation and differentiation of stem cells are influenced by interaction between cells and their microenvironment. Extracelluar matrix (ECM) forms the chunk of niche with its components, which is remodelled by the cellular activity. The remodelling and change of ECM components have an impact on cellular activity. ECM plays a vital role in the uncontrolled proliferation of cancer cells. In the present study, we have examined the proliferative ability of mouse embryonic stem cells (C3H10 T1/2 clone 8) grown on cell-free ECM isolated from $\mathrm{KB}$ and MCF-7 cancer cell line cultures, separately, using standard ammonium hydroxide method. The texture of ECM was characterized from images captured with inverted microscope and scanning electron microscope (SEM). The growth of C3H10 T1/2 clone 8 cells over KB-ECM and MCF-ECM was monitored up to $192 \mathrm{~h}$ and the doubling time was estimated. KB-ECM promoted growth rate by reducing the doubling time from 23 to $14 \mathrm{~h}$, whereas MCF-ECM prolonged the lifetime of stem cells by extending the log phase of growth. The surface topography of KBECM under SEM showed rough, irregular and meshlike structure compared to MCF-7-derived ECM. This may account for enhanced growth rate of stem cells. The findings underscore the relevance of modifications to scale-up the generation of stem cells for use in regenerative medicine. Further studies are required with different sources of stem cells grown on modified components of ECM to identify the appropriate ECM.

Keywords: Cancer cell lines, extracellular matrix, regenerative medicine, stem cells, tissue engineering.

TISSUE engineering basically requires cells, molecules and materials. With these components, organs are engineered in the laboratory before being implanted at the site of damage, or by treating with stem cells or materials or molecules, or the triad together, to coax regeneration of the damaged tissue in situ. Indeed, the triad needs to be engineered individually before proceeding for tissue

*For correspondence. (e-mail: koyelig@gmail.com) engineering. Every tissue has a unique composition of extracelluar matrix (ECM) and topology formed during development through a dynamic reciprocal interaction between cells and their evolving new environment. ECM components include structural proteins such as collagen, laminin, fibronectin, elastin, etc. secreted growth factors and plasma membrane-associated adhesion proteins. The composition is not static, but undergoes dynamic remodelling through deposition, degradation and modification varying from tissue to tissue ${ }^{1-3}$. Biomechanical properties of ECM regulate cell behaviours, including cell-fate determination, differentiation and tissue function ${ }^{4,5}$. The remodelled ECM by cells in turn modifies the behaviour of cells ${ }^{6}$, which allows cells and tissues to swiftly adapt to their environment ${ }^{7}$. The ECM of tumour tissue is stiffer than normal tissue ${ }^{8}$. An increase in collagen deposition or ECM stiffness-associated integrin signalling can promote cell survival and proliferation ${ }^{9,10}$. The paracrine signalling due to cell-cell interactions in the stem cell niche has been shown in multiple organ systems ${ }^{11,12}$. Bioengineers have recreated the ECM niche with its biomechanical cues in vitro ${ }^{13}$. The mouse embryonic stem cell-derived embryoid bodies cultured in polymer networks made of collagen, fibronectin or laminin show significantly different differentiation and viability depending on the composition of ECM ${ }^{14}$. The dynamic nature ECM in particular, and the niche as a whole, could not only alter the growth and differentiation of stem cells, but also make it a therapeutic target for regenerative medicine. The regulated stiffness of ECM constructs as well as engineered artificial ECMs form the potential tool in maintaining the stemness and lineage commitment of the stem cells ${ }^{15}$. Specific lineage-engineered mesenchymal stem cells are shown to complete their fate when grown on a cell-secreted, decellularized $\mathrm{ECM}^{16}$. ECM complexity can enhance cell response and such an ECM can be used to coat implantable biomaterials to achieve lineage attainment ${ }^{17,18}$. Recently, it has been shown that the use of $3 \mathrm{D}$ cultures mimics in vivo cellular behaviour with biochemical and biomechanical cues for cellular proliferation, differentiation, migration, adhesion and gene expression $^{19}$. Thus, artificial ECM constructs, through 
physical and molecular interactions, enable direct control of stem cell behaviour.

The lack of adequate number of stem cells limits their use for certain tissue engineering applications; however, the ability of stem cells to expand in culture retaining the potential to differentiate into specific lineages can address this issue. Further, the selection of suitable ECM composition can facilitate the expansion of stem cells on a large scale. In the present study, we examine the potential of ECM secreted by cancer cells in supporting the proliferation of mouse embryonic stem cell line, C3H10. As cancer microenvironment is well known to support abundant proliferation of cancer cells, it is expected to have such an expansion of stem cells grown on ECM derived from cancer cells, which can meet the increasing demand for stem cells in tissue engineering and regenerative medicine.

\section{Materials and methods}

\section{Materials}

KB cell line, C3H10 T1/2 clone 8 cell line and MCF-7 cell line were procured from National Centre for Cell Science (NCCS), Pune. Dulbecco's Modified Essential Medium (DMEM) from HiMedia (India), Hank's base salt solution (HBSS) from Sigma (USA), while fetal bovine serum (FBS), antibiotic solution and trypsin were purchased from Gibco (USA). Other chemicals were procured locally.

\section{Cell culture}

All the cell lines were cultured with DMEM supplemented with $10 \%$ FBS and $1 \%$ antibiotic solution, according to Ghosh and Girigoswami ${ }^{20}$. The cells were incubated at $37^{\circ} \mathrm{C}$ in a $\mathrm{CO}_{2}$ incubator with humidified atmosphere. $\mathrm{C} 3 \mathrm{H} 10 \mathrm{~T} 1 / 2$ clone $8, \mathrm{MCF}-7$ cells and $\mathrm{KB}$ cells were received from NCCS, Pune and kept in the incubator at $37^{\circ} \mathrm{C}$ for $48 \mathrm{~h}$. After $48 \mathrm{~h}$, the cells were trypsinized using $3 \times$ trypsin for $\mathrm{C} 3 \mathrm{H} 10$ and $1 \times$ trypsin for MCF-7 and KB cells and subcultured. The subcultured cells were maintained according to the procedure described by Ghosh and Girigoswami ${ }^{20}$.

\section{Isolation of ECM}

ECM was isolated from $\mathrm{KB}$ and MCF-7 cells. After equal cell number seeding in control and ECM-marked plates, the ECM was isolated using ammonium hydroxide method $^{21}$, with slight modifications. One millilitre of ammonium hydroxide was added to the cells after washing them with sterile HBSS and shaken every $1 \mathrm{~min}$ for further $5 \mathrm{~min}$. HBSS was added to stop the ammonium hydroxide reaction and the plates were washed with HBSS to remove the residual alkali. ECM isolated from
$\mathrm{KB}$ cells (KB-ECM) and MCF-7 cells (MCF-ECM) respectively, was sterilized using $70 \%$ ethanol, air-dried under sterile conditions and $\mathrm{C} 3 \mathrm{H} 10 \mathrm{~T} 1 / 2$ clone 8 cells were cultured above the ECM after appropriate dilution. For characterization of KB-ECM and MCF-ECM, the KB and MCF-7 cells were cultured on glass coverslips and processed for ECM isolation using an ammonium hydroxide treatment method. Coverslips of the same size were used for all the experiments.

\section{Characterization of the isolated ECM}

The KB-ECM showed enhanced doubling time of the C3H10 T1/2 clone 8 cells. Thus, we analysed the morphological structure of KB-ECM according to Girigoswami et $a l .^{22}$. Samples for SEM were prepared by culturing the KB cells on sterile glass coverslips placed inside a $35 \mathrm{~mm}$ dish and isolating the ECM using the method described above. The coverslips were air-dried and processed for SEM analysis.

\section{Cell functional assay}

For cell functional assays, C3H10 T1/2 clone 8 cells were cultured over KB-ECM and MCF-ECM as well as in polystyrene-coated culture plates (without any ECM) as control. The procedure followed for growth curve calculation of $\mathrm{C} 3 \mathrm{H} 10 \mathrm{~T} 1 / 2$ clone 8 cells was according to Girigoswami and Ghosh ${ }^{23}$. Equal number of C3H10 T1/2 clone 8 cells was seeded in the KB-ECM and MCF-ECM plates and in normal plates (control), incubated in $\mathrm{CO}_{2}$ incubator and after every $24 \mathrm{~h}$ the cells were observed under an inverted microscope, and images were captured using a CCD camera. Further, the cells were trypsinized, suspended in HBSS and counted using a haemocytometer. The doubling of cells was monitored for $192 \mathrm{~h}$.

\section{Results}

\section{Cell culture}

Three cell lines, namely $\mathrm{C} 3 \mathrm{H} 10 \mathrm{~T} 1 / 2$ clone 8 cell line (fibroblast-like cells isolated from rat embryo), $\mathrm{KB}$ cell line (oral cancer cell line), and MCF-7 cell line (breast cancer cell line) were selected for the present study. MCF-7 and KB cells were used as two different types of cancer cells for the isolation of ECM on which $\mathrm{C} 3 \mathrm{H} 10$ T1/2 clone 8 cells were grown. Supplementary Figure 1 shows the typical snapshots of the cell lines used and visualized under inverted microscope.

\section{ECM characterization}

The ECM was isolated from KB and MCF-7 cells. To screen the absence of any live cells in the ECM deposited 

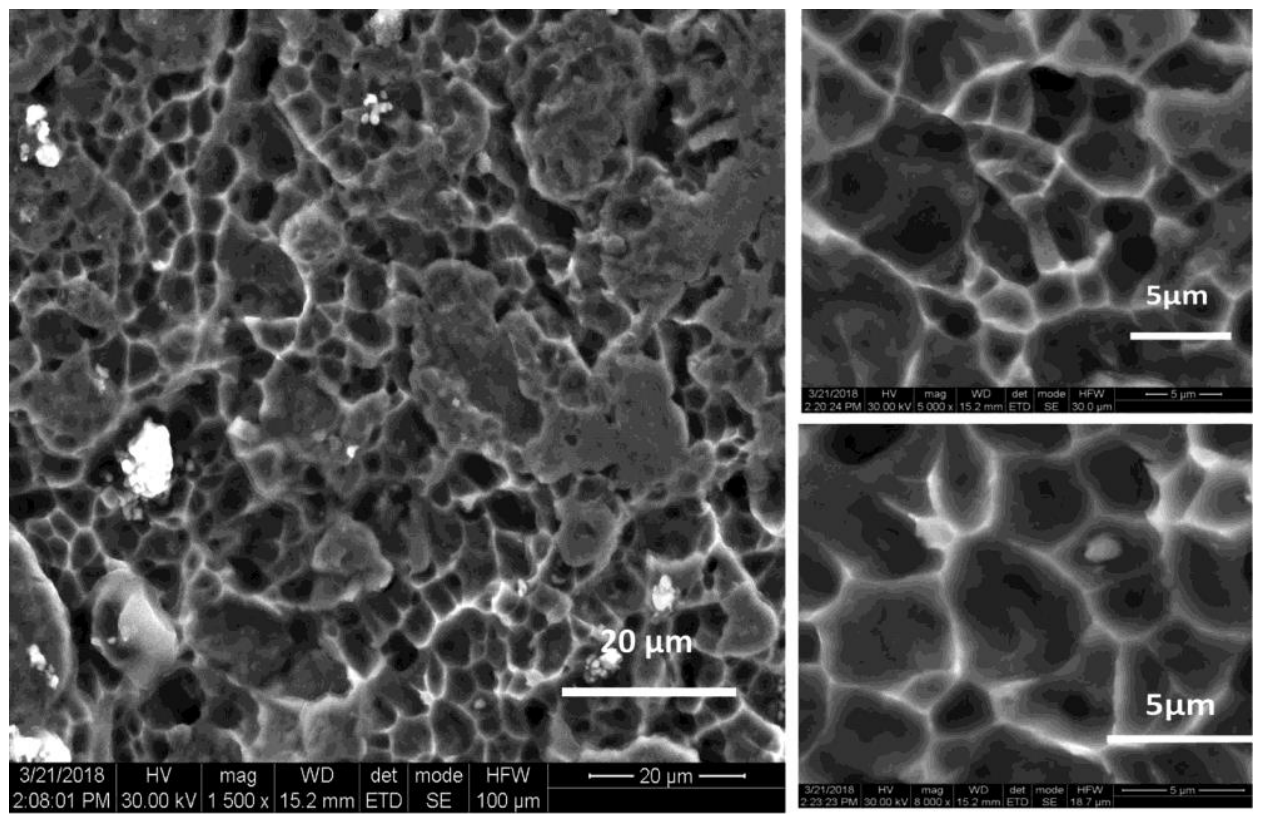

Figure 1. Scanning electron microscope image of the KB-extracelluar matrix (ECM) at different magnifications

in tissue-culture dishes, they were observed under inverted microscope. Supplementary Figure 2 shows the inverted microscope images of KB-ECM, MCF-ECM and normal polystyrene-coated tissue culture dish (control). The topography of control (polystyrene-coated) plates showed small granular morphology, KB-ECM showed striated morphology and MCF-ECM revealed a bumpy surface.

To study the surface morphology of KB-ECM with SEM, we have cultured KB cells over glass coverslips (Supplementary Figure 3). The SEM images of KB-ECM isolated from the KB cells grown by the glass coverslips were captured at different magnifications (Figure 1).

\section{C3H10 functional assay on KB-ECM and MCF-ECM}

C3H10 T1/2 clone 8 cells were cultured over KB-ECM or MCF-ECM and control polystyrene-coated tissue culture dishes with equal initial cell count $\left(5 \times 10^{4}\right.$ cells/plate $)$ for both sets of plates. The cells were observed under inverted microscope. Figure $2 a$ and $b$ show typical photographs of $\mathrm{C} 3 \mathrm{H} 10 \mathrm{~T} 1 / 2$ clone 8 cells grown over KB-ECM and MCF-ECM respectively, at different time intervals after culture.

\section{Growth curve of $C 3 H 10$ cells on KB-ECM and $M C F-E C M$}

After growing the $\mathrm{C} 3 \mathrm{H} 10 \mathrm{~T} 1 / 2$ clone 8 cells over $\mathrm{KB}$ ECM or MCF-ECM and tissue culture plates (control), they were trypsinized at different time intervals, diluted in HBSS and counted using a haemocytometer. The number of cells versus time was plotted to obtain the growth curve of C3H10 T1/2 clone 8 cells on KB-ECM or MCF$\mathrm{ECM}$ and control (polystyrene surface) (Figure $3 a$ and $b$ respectively). The doubling time of $\mathrm{C} 3 \mathrm{H} 10 \mathrm{~T} 1 / 2$ clone 8 cells on the control surface was $23 \mathrm{~h}$, whereas in case of KB-ECM it reduced to $14 \mathrm{~h}$. Observations till $192 \mathrm{~h}$ showed that the cells nearly reached $100 \%$ confluency and we could not continue with further observations. The C3H10 T1/2 clone 8 cells over KB-ECM did not show any lag phase in growth; rather they started by attaching and multiplying within $24 \mathrm{~h}$. The $\mathrm{C} 3 \mathrm{H} 10 \mathrm{~T} 1 / 2$ clone 8 cells did not show any difference in doubling time when grown on MCF-ECM compared to control surface, and the doubling time was found to be $23 \mathrm{~h}$. There was extension in growth observed after $144 \mathrm{~h}$ showing a prolonged log phase which was not observed for control plates, where the cells entered the stationary phase after $144 \mathrm{~h}$.

\section{Calculation of doubling time}

The doubling time of $\mathrm{C} 3 \mathrm{H} 10 \mathrm{~T} 1 / 2$ clone 8 cells grown on KB-ECM and MCF-ECM was calculated using data obtained from the growth curves (Figure $3 a$ and $b$ respectively). The formula used for calculating the doubling time is as follows

$$
\mathrm{gr}=\ln \left(N_{(t)} / N_{(0)}\right) / t,
$$

where $N_{(t)}$ is the number of cells at time $t, N_{(0)}$ the number of cells at time 0 , gr the growth rate and $t$ is the time (h). Doubling time $=\ln 2 / \mathrm{gr}$. 
RESEARCH ARTICLES

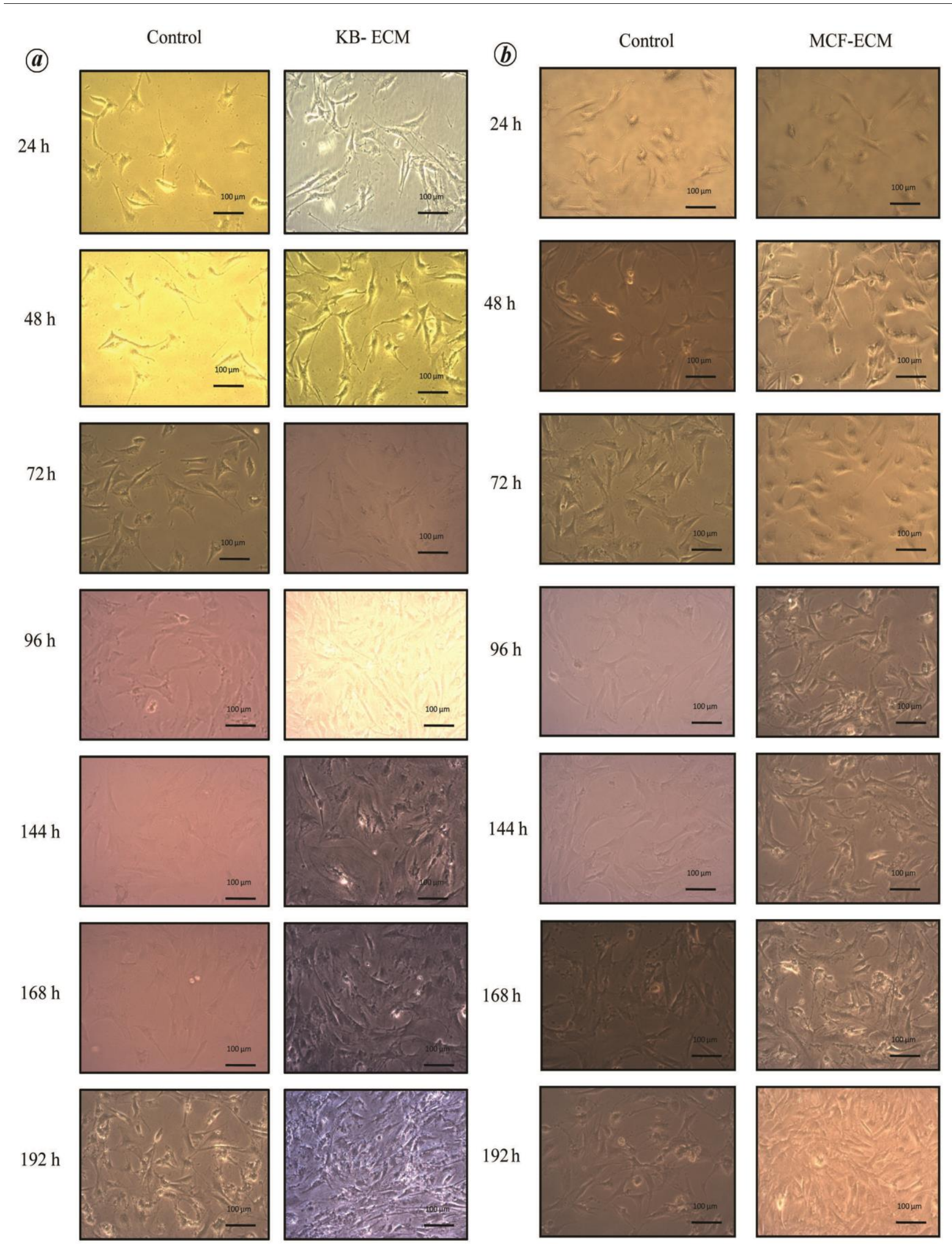

Figure 2. Study of C3H10 T1/2 clone 8 functional assay over (a) KB-ECM and (b) MCF-ECM compared with control (polystyrene surface) at different incubation periods. Scale bar denotes $100 \mu \mathrm{m}$. 

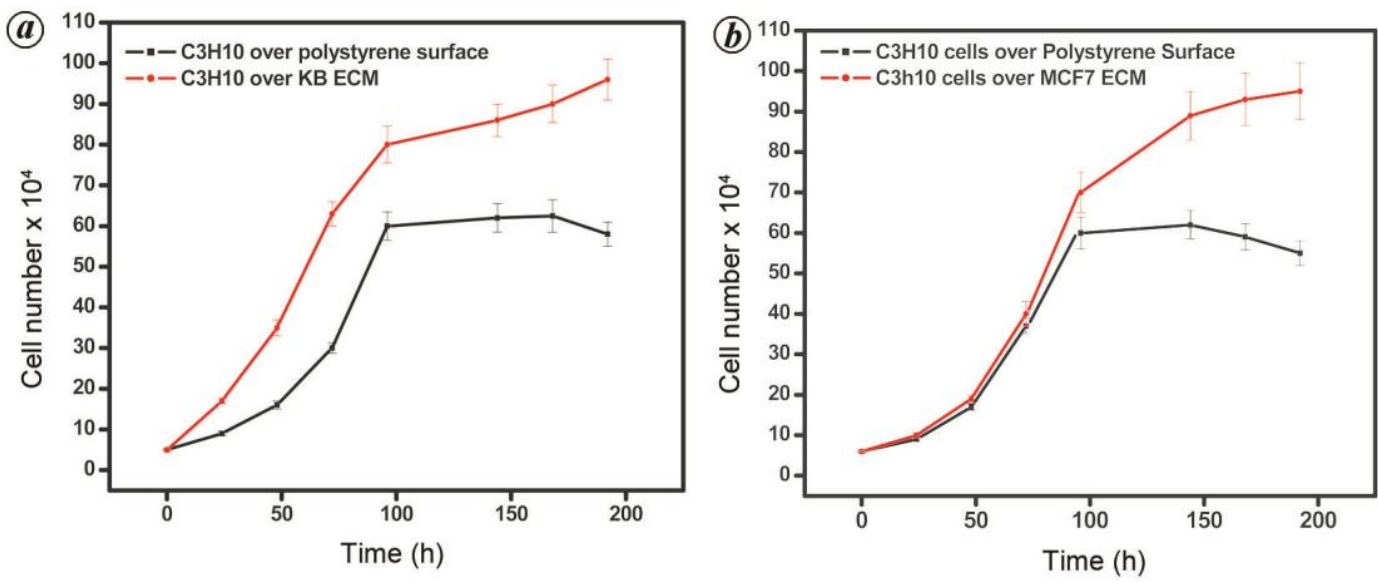

Figure 3. Growth curve of C3H10 T1/2 clone 8 cells over (a) KB-ECM (red line) and control (polystyrene surface; black line) and (b) MCF-ECM (red line) and control (polystyrene surface; black line).

\section{Discussion}

Stem cell-based therapy is one of the regenerative strategies used to replace damaged tissues and organs, or to induce the self-healing capacity. Even though stem cells are present in every tissue, their number is relatively ${ }_{10}{ }^{24}$. However, most of the clinical protocols require $20-100 \times 10^{6}$ stem cells. The high number of cells can be expanded in vitro using appropriate scaffolds or biomaterials ${ }^{25}$. The scaffolds can be of synthetic or natural materials, including ECM components that provide biological cues to cells ${ }^{26-28}$. ECM components are shown to play a significant role in breast cancer progression and metastasis $^{29}$. In breast cancer, significant changes occur in ECM composition. Interestingly, ECM in breast cancer exhibits high similarity with the matrix in tissues undergoing wound healing or remodelling ${ }^{29,30}$. Studies have suggested that cancer can be controlled to produce normal cell types by changing the microenvironment, or can be reverted into normal phenotype by altering the signalling of the matrix ${ }^{31}$. The proteins of ECM are multifunctional aiding cell migration, morphogenesis, proliferation, differentiation and apoptosis ${ }^{32}$. Increased production of collagen, laminin and elastin also results in elevated stiffness of tumour compared to the surrounding normal tissue $^{33}$. The surface topography and matrix substrate geometry can also control the cell-adhesion integrin receptors and initiate a range of responses ${ }^{34}$. In the present study, we examined the impact of ECM from cancer cells on the proliferation of stem cells.

We have successfully cultured three types of cells, namely C3H10 T1/2 clone 8, KB and MCF-7 (Supplementary Figure 1). The cells were healthy and attached well to the plate surface. The isolation of ECM was done using standard protocol and ammonium hydroxide treatment did not show any residual cells with ECM coated on the plate (Supplementary Figure 2). The surface of control (polystyrene-coated) plates showed small granular morphology, KB-ECM showed striated morphology, whereas MCF-ECM revealed a bumpy surface, under inverted microscope. The morphological features of C3H10 T1/2 clone 8 cells grown over KB-ECM established proper attachment and spreading, compared to cells seeded over polystyrene surface. SEM images of KB-ECM (Supplementary Figure 3 and Figure 1) showed rough, irregular, mesh-like structure, which further supported the prevalence of a conducive environment.

The decrease $(14 \mathrm{~h})$ in doubling time of stem cells (C3H10 T1/2 clone 8) seeded over KB-ECM, compared to control and MCF-ECM substrate $(23 \mathrm{~h})$, underscores the facilitatory effects of KB-ECM on stem cell proliferation (Figure $3 a$ ). The growth curve of $\mathrm{C} 3 \mathrm{H} 10 \mathrm{~T} 1 / 2$ clone 8 cells grown over KB-ECM did not show any lag phase. Instead, the cells directly got into log phase. Moreover, at each point of time, the number of cells was significantly higher in KB-ECM plates compared to control $(P \leq 0.05)$ with doubling time, $14 \mathrm{~h}$ versus $23 \mathrm{~h}$. In short, KB-ECM seems to provide the appropriate environment that promotes higher growth. The absence of such growth in cells seeded over MCF-ECM may be due to the possible difference in ECM components between these two sources. This difference may also have been contributed by differential interaction between stem cells and these substrates. Interestingly, stem cells grown on MCF-ECM that maintained a lag phase up to $96 \mathrm{~h}$, as in the case of control, registered an increase in growth rate after $144 \mathrm{~h}$ of culture when compared to control cells. This may be due to the delayed remodelling of conducive ECM components that support cell proliferation. Recently, bone marrow-derived mesenchymal stem cells (BMSC) and adipose-derived stem cells grown on type-IV collagen or fibronectin-coated plates proliferated well, except on laminin-coated polystyrene culture plates ${ }^{35}$. Mesenchymal stem cells are shown to produce ECM proteins, such as 
collagen type I and III, laminin, vimentin and osteonectin, which they seem to modify for their differentiation through interaction with the matrix ${ }^{36}$. Lanfer et al. ${ }^{37}$ have reported that cells grown on standard polystyrene culture vessels lose their original organization found in physiological conditions. Considering these findings, it seems reasonable that characterizing the ECM components in these substrates can explain this discrepancy.

In the present study, the $\mathrm{C} 3 \mathrm{H} 10 \mathrm{~T} 1 / 2$ clone 8 used belongs to the aneuploid type of cells which are generally considered as post-mitotic. According to Alberts et al. ${ }^{38}$, the aneuploid cells can proliferate at a slow rate. More than $90 \%$ of solid tumours are aneuploid, and tumour cells are known to have uncontrolled rate of proliferation. In agreement with these reports, we also observed an increase in the number of stem cells grown over ECM derived from cancer cells. Generally, stem cells are known to have self-renewal property that must have been facilitated by ECM used here, in spite of their aneuploid nature. Future studies are proposed with different types of stem cells. Our preliminary study points to the facilitatory role of cancer cell-derived ECM on stem-cell proliferation.

\section{Conclusion}

In conclusion, the cancer cell ECM isolated from two different cell lines contributes in a differential manner to the growth rate of stem cells. KB-ECM enhances growth rate, whereas MCF-ECM induces enhanced log phase in the stem cells. Further studies on the characterization of cancer cell-derived ECM are essential to clearly demarcate the role of endogenous biomaterials.

Statement of ethics: The cell lines used in this study were obtained from NCCS, Pune. These cell lines do not involve human embryonic stem cells or embryonic germ cells.

Disclosure statement: The authors declare no conflict of interest.

1. Haque, M. A., Nagaoka, M., Hexig, B. and Akaike, T., Artificial extracellular matrix for embryonic stem cell cultures: a new frontier of nanobiomaterials. Sci. Technol. Adv. Mater., 2010, 11, 1-10.

2. Lu, P., Weaver, V. M. and Werb, Z., The extracellular matrix: a dynamic niche in cancer progression. J. Cell Biol., 2012, 196, 395-406.

3. Ahmed, M. and Ffrench-Constant, C., Extracellular matrix regulation of stem cell behavior. Curr. Stem Cell Rep., 2016, 2, 197206

4. Lutolf, M. P., Gilbert, P. M. and Blau, H. M., Designing materials to direct stem-cell fate. Nature, 2009, 462(7272), 433-441.

5. Gilbert, P. M. et al., Substrate elasticity regulates skeletal muscle stem cell self-renewal in culture. Science, 2010, 329, 1078-1081.

6. Butcher, D., Alliston, T. and Weaver, V., A tense situation: forcing tumour progression. Nature Rev. Cancer, 2009, 9, 108-122.
7. Samuel, M. S. et al., Actomyosin-mediated cellular tension drives increased tissue stiffness and $\beta$-catenin activation to induce epidermal hyperplasia and tumor growth. Cancer Cell, 2011, 19, 776-791.

8. Lopez, J. I., Kang, I., You, W.-K., McDonald, D. M. and Weaver, V. M., In situ force mapping of mammary gland transformation. Integr. Biol., 2011, 3, 910-921.

9. Wozniak, M. A., Desai, R., Solski, P. A., Der, C. J. and Keely, P. J., ROCK-generated contractility regulates breast epithelial cell differentiation in response to the physical properties of a threedimensional collagen matrix. J. Cell Biol., 2003, 163, 583-595.

10. Paszek, M. J. et al., Tensional homeostasis and the malignant phenotype. Cancer Cell, 2005, 8, 241-254.

11. Kronenberg, H. M., PTH regulates the hematopoietic stem cell niche in bone. Adv. Exp. Med. Biol., 2007, 602, 57-60.

12. Kasper, S., Exploring the origins of the normal prostate and prostate cancer stem cell. Stem Cell Rev., 2008, 4, 193-201.

13. Kim, D. H., Wong, P. K., Park, J., Levchenko, A. and Sun, Y., Microengineered platforms for cell mechanobiology. Аnпи. Rev. Biomed. Eng., 2009, 11, 203-233.

14. Battista, S. et al., The effect of matrix composition of 3D constructs on embryonic stem cell differentiation. Biomaterials, 2005, 26, 6194-6207.

15. Handorf, A. M., Zhou, Y., Halanski, M. A. and Li, W. J., Tissue stiffness dictates development, homeostasis, and disease progression. Organogenesis, 2015, 11(1), 1-15.

16. Hoch, A. I., Mittal, V., Mitra, D., Vollmer, N., Zikry, C. A. and Leach, J. K., Cell-secreted matrices perpetuate the bone-forming phenotype of differentiated mesenchymal stem cells. Biomaterials, 2016, 74, 178-187.

17. Decaris, M. L., Binder, B. Y., Soicher, M. A., Bhat, A. and Leach, J. K., Cell-derived matrix coatings for polymeric scaffolds. Tissue Eng., 2012, 18(19-20), 2148-2157.

18. Harvestine, J. N., Vollmer, N. L., Ho, S. S., Zikry, C. A., Lee, M. A. and Leach, J. K., Extracellular matrix-coated composite scaffolds promote mesenchymal stem cell persistence and osteogenesis. Biomacromolecules, 2016, 17(11), 3524-3531.

19. Nair, G. G. et al., Recapitulating endocrine cell clustering in culture promotes maturation of human stem cell-derived $\beta$ cells. Nature Cell Biol., 2019, 21, 263-274.

20. Ghosh, R. and Girigoswami, K., NADH dehydrogenase subunits are overexpressed in cells exposed repeatedly to $\mathrm{H}_{2} \mathrm{O}_{2}$. Mutat. Res., 2008, 638, 210-215.

21. Hellewell, A. L., Rosini, S. and Adams, J. C., A rapid, scalable method for the isolation, functional study and analysis of cellderived extracellular matrix. J. Vis. Exp., 2017, 119, 55051

22. Girigoswami, K., Ku, S. H., Ryu, J. and Park, C. B., A synthetic amyloid lawn system for high-throughput analysis of amyloid toxicity and drug screening. Biomaterials, 2008, 29(18), 2813-2819.

23. Girigoswami, K. B. and Ghosh, R., Response to $\gamma$-irradiation in V79 cells conditioned by repeated treatment with low doses of hydrogen peroxide. Radiat. Environ. Biophys., 2005, 44(2), 131137.

24. Caplan, A. I., Why are MSCs therapeutic? New data: new insight J. Pathol., 2009, 217(2), 318-324.

25. Groeber, F., Holeiter, M., Hampel, M., Hinderer, S. and SchenkeLayland, K., Skin tissue engineering - in vivo and in vitro applications. Adv. Drug Deliv. Rev., 2011, 63, 352-366.

26. Hinderer, S., Seifert, J., Votteler, M., Shen, N., Rheinlaender, J., Schäffer, T. E. and Schenke-Layland, K., Engineering of a biofunctionalized hybrid off-the-shelf heart valve. Biomaterials, 2014, 35, 2130-2139.

27. Wang, H. et al., Promotion of cardiac differentiation of brown adipose derived stem cells by chitosan hydrogel for repair after myocardial infarction. Biomaterials, 2014, 35, 3986-3998.

28. Monaghan, M., Browne, S., Schenke-Layland, K. and Pandit, A., A collagen-based scaffold delivering exogenous microRNA-29B 
to modulate extracellular matrix remodelling. Mol. Ther., 2014, 22(4), 786-796.

29. Oskarsson, T., Extracellular matrix components in breast cancer progression and metastasis. Breast, 2013, 22, S66-S72.

30. Lu, P., Takai, K., Weaver, V. M. and Werb, Z., Extracellular matrix degradation and remodeling in development and disease. Cold Spring Harbor Perspect. Biol., 2011, 3(12), a005058.

31. Nelson, C. M. and Bissell, M. J., Of extracellular matrix, scaffolds, and signaling: tissue architecture regulates development, homeostasis, and cancer. Annu. Rev. Cell Dev. Biol., 2006, 22, 287-309.

32. Rozario, T. and DeSimone, D. W., The extracellular matrix in development and morphogenesis: a dynamic view. Dev. Biol., 2010, 341(1), 126-140.

33. Chaudhuri, O. et al., Extracellular matrix stiffness and composition jointly regulate the induction of malignant phenotypes in mammary epithelium. Nature Mater., 2014, 13(10), 970978.

34. Dalby, M. J., Gadegaard, N. and Oreffo, R. O., Harnessing nanotopography and integrin-matrix interactions to influence stem cell fate. Nature Mater., 2014, 13(6), 558-569.

35. Anna, B., Dorota, P. and Krzysztof, R., Influence of extracellular matrix on the proliferation and adhesion properties of stem cells derived from different sources. Eur. J. Biol. Res., 2017, 7(3), 165171.

36. Lai, Y. et al., Reconstitution of marrow-derived extracellular matrix ex vivo: a robust culture system for expanding large-scale highly functional human mesenchymal stem cells. Stem Cells Dev., 2010, 19, 1095-1107.

37. Lanfer, B., Seib, F. P., Freudenberg, U., Stamov, D., Bley, T., Bornhäuser, M. and Werner, C., The growth and differentiation of mesenchymal stem and progenitor cells cultured on aligned collagen matrices. Biomaterials, 2009, 30, 5950-5958.

38. Alberts, B., Bray, D., Lewis, J., Raff, M., Roberts, K. and Watson, J. D., Molecular Biology of the Cell (Third edition), Garland Publishing, New York, USA, 1994, p. 1361.

ACKNOWLEDGEMENT. We thank the Chettinad Academy of Research and Education, Kelambakkam for infrastructural support.

Received 6 March 2020; revised accepted 4 February 2021

doi: $10.18520 / \mathrm{cs} / \mathrm{v} 120 / \mathrm{i} 10 / 1616-1622$ 